respiratory symptoms. However, quantitative exposures to these products are not well-characterized. The objective of this study was to characterize exposures to cleaning and disinfecting chemicals in healthcare occupations.

Methods Exposure assessments were conducted at five hospitals targeted 14 healthcare occupations. Mobile-area and personal real-time and time-integrated air monitoring was conducted for volatile organic compounds (VOCs), and 14 specific VOCs were quantified, including total VOCs (TVOCs). Quaternary ammonium compounds (QACs) were quantified at one hospital. Exposure data were summarized by occupation and geometric means (GMs) and geometric standard deviations (GSDs) were calculated. GM exposure estimates for product-application tasks were obtained from linear regression models.

Results The GMs for TVOCs were highest among nursing assistants, licensed practical nurses, and medical equipment preparers (range: 4367-2142 ppb), followed by respiratory therapists, pharmacy technicians, registered nurses, housekeepers, floor strippers/waxers, and dental assistants (range: 2120$1565 \mathrm{ppb})$; GSDs varied from 1.06 to 9.01. GMs for selected VOCs were: ethanol (1.54-2594 ppb), acetone (18.5-70.6 $\mathrm{ppb}$ ), chloroform (0.09-0.57 ppb), $\alpha$-pinene (0.04-0.19 ppb), and d-limonene (0.12-4.23 ppb). Real-time TVOC GMs were the highest for the product-application tasks of using skin wipes containing QACs, using enzymatic cleaners, using glasscleaning products, cleaning instruments with high-level disinfectants, and using detergents to clean surfaces (2091-1049 $\mathrm{ppb}$ ). Specific VOC levels were strongly associated with product-application tasks; using products containing bleach or QACs predicted levels of chloroform and d-limonene, respectively. Concentrations of QACs were low in short-duration $\left(0.23-1.5 \mu \mathrm{g} / \mathrm{m}^{3}\right)$ and full-shift $\left(0.006-0.028 \mu \mathrm{g} / \mathrm{m}^{3}\right)$ area air samples.

Conclusions Exposure levels of total and specific VOCs varied by occupations and tasks. These estimates can be used to generate a job-task exposure matrix for use in epidemiologic studies.

\section{S-66 COVID-19 AND THE WORKPLACE}

'Lode Godderis, Jean-Baptist du Prel, Damien McElvenny, Ingrid Sivesind Mehlum, Susan Peters, Vivi Schlünssen, Kurt Straif, Michelle Turner. 'IDEWE, Belgium and KU Leuven, Belgium

\subsection{6/OEM-2021-EPI.399}

The SARS-CoV-2 virus pandemics has raised several challenges at the workplace. Within the omega-net COVID-19 taskforce, we developed standardized COVID-19 questionnaires for occupational research, a multi-country COVID-19 Job Exposure Matrix, and research on COVID-19 as an occupational disease. The compiled questionnaire resource covers all key aspects of the COVID-19 pandemic, including: COVID-19 diagnosis \&amp; prevention, Health and demographic, Use of personal protective equipment, Health effects, Work-related effects, Financial effects, Work-based risk factors, Psychosocial risk factors, Lifestyle risk factors, and Personal evaluation of the impact of COVID-19. For each of the domains additional questions are available. A second questionnaire (in a short and along version) focusses on occupational risk factors for SARS-CoV-2 infection and COVID-19 disease. The questionnaires are available online at https://omeganetcohorts.eu/news/covid19-questionnairesomeganet/. The JEM was developed by experts from three European countries (Denmark, the Netherlands, UK), who defined the relevant exposure and workplace characteristics with regard to the possible exposure to SARS-COV 2 infection. The C19-JEM contains four determinants of transmission risk (number of people, type of people, indirect contact and location), two mitigation measures (social distance and face covering), and two social factors (income insecurity and migrant workers). Finally, we developed and piloted a questionnaire on COVID-19 as occupational disease, which provide data on 1) COVID-19 as occupational disease or injury, 2) criteria for recognition and compensation regarding exposure, disease, role of use of PPE and of competing non-occupational exposure; and 3) what can be compensated. Results are available from 36 largely European countries. Through the development and implementation of tools we not only provide instruments, but also insights on the occupational risks and diseases in relation to SARS-COV 2 .

\section{S-73 ARE EARLY WORKING LIFE PATTERNS RELATED TO THE COURSE OF FUTURE SICKNESS ABSENCE DUE TO COMMON MENTAL DISORDERS?}

${ }^{1}$ Amaya Ayala-Garcia, Laura Serra, Monica Ubalde-Lopez. ${ }^{1}$ Universitat Pompeu Fabra, Spain

\subsection{6/OEM-2021-EPI.400}

Introduction In recent decades, the labour market has evolved into temporary employment, part-time work, and long-term unemployment, especially affecting young population and women. Transitions between states during working life could affect mental health.

Objectives To assess the relationship between early working life (EWL) patterns and the future course of sickness absence (SA) due to mental disorders.

Methods Cohort study of a working sample between 18 and 28 years of age, residents in Catalonia, with at least one episode of SA due to a mental disorder between 2012 and 2014. Reconstruction of individual working life trajectories previous to the SA were carried out by sequence analysis based on four work-related states (permanent, temporary contract, unemployment and without social security coverage). By optimal matching, patterns were identified out of clustering similar working trajectories. Identification of SA trajectories were made by latent class growth modelling analysis. Adjusted multinomial logistic regression models were applied to assess the relationship between early working life patterns and SA trajectories.

Results Among men, fluctuating (aOR 1.25[95\% CI: $0.57-$ 2.74]) or delayed (aOR 1.79 [95\% CI: 0.59-5.41]) entry into the labour market showed a trend towards a middle stable accumulation of SA days. In women, an increasing permanent and fluctuating employment pattern (aOR 2.41 [95\% CI: 1.01-5.75]) at the beginning of their EWL was related to a decreased accumulation of future days on SA due to mental disorders (aOR 2.08 [95\% CI: 1.18-3.66]) rather than to a low stable trajectory.

Conclusions An unstable early working life built on a high number of transitions between temporary contracts, unemployment and lack of social security coverage states is related to a future worse SA course due to mental diagnosis. 